\title{
Cancer control: a reminder of the need for a balanced approach between prevention and treatment
}

\author{
Christopher P. Wild ${ }^{7}$
}

The recent World Cancer Report 2014 highlighted a number of critical points relevant to cancer control, encompassing occurrence, causes, molecular and cellular mechanisms and early detection, prevention and treatment of the disease (1). There are clear pointers from the body of knowledge in this Report which should contribute to the evidence base for cancer control.

Cancer is a growing problem. The GLOBOCAN estimates for 2012 were 14.1 million new cases and 8.2 million deaths worldwide. However, the population demographics of ageing and growth will result in around $40 \%$ more new cancer patients annually by 2025. In the countries of the World Health Organization (WHO) Eastern Mediterranean Region (EMR), the estimated increase is from 555000 to 819000 new cases over this period, an increase of $68 \%$ in just over a decade (2). In terms of disability-adjusted life years, the low- and middle-income countries are already heavily affected because the common cancers (e.g. liver, stomach, cervix) occur at younger ages and have poorer survival rates $(3,4)$. Indeed, cancer is one of the most common causes of death in the Arab world, ranking third in Oman, for example (5). There is a risk of under-prioritizing cancer, however, because global burden analyses commonly present neoplasms separately for each organ, and the result is that cancer does not appear in the
20 most common causes of death in EMR (6).

Whilst cancer is a universal problem, it is not a uniform one. The patterns and related causes differ markedly by geographical region and level of human development. This heterogeneity has important implications. For example, chronic infections are linked to 1 in 3 cancers in sub-Saharan Africa but only 1 in 30 in Australia (7). Clearly this must translate into different priorities for national cancer control plans. In the EMR countries the 5 most common cancers among men are lung, bladder, liver, prostate and colorectum, and among women are breast, colorectum, cervix, ovary and non-Hodgkin lymphoma. In contrast to men, for whom the incidence of these 5 cancers is quite similar, the pattern in women is dominated by breast cancer, with 1 in 3 of all new cancers being breast cancer. In addition, the importance of individual cancer sites varies substantially across EMR countries, e.g. more than 3-fold for cancer of the lung and breast.

It is important to note that the above estimates are frequently based on limited national data, circumstances under which it is difficult for countries to make rational cancer control plans. The need to prioritize support to population-based cancer registries has led the International Agency for Research on Cancer and WHO to develop the multi-partner project the Global Initiative for Cancer Registries
(8). In the first instance, priority should be given to developing high-quality cancer registries covering populations in defined geographical areas within countries (e.g. a district or the capital region), rather than attempting to implement registration covering the entire country. Initiating national cancer registration is usually an unrealistic prospect in low- and middle-income countries; either it is technically unfeasible or the cost involved greatly outweighs the additional benefits obtained from registration of a sample of the population (9).

What priorities should be set to meet this growing cancer challenge? Even in the highest income countries the spiralling costs of cancer treatment and care are difficult to sustain. In many countries access to therapy remains limited and personalized medicine is far off. It is implausible for the world to treat its way out of cancer. A high priority must be placed on prevention and early detection of the disease, particularly in low and middle-income countries.

Identification of risk factors is the foundation of cancer prevention, and much is already known about this, with typical estimates of $30 \%-50 \%$ of cancers being preventable, based on current knowledge (10). The high rates of smoking in EMR countries only serve to emphasize that the first priority must be tobacco control, notably implementation of the WHO Framework Convention on Tobacco 
Control. Other priorities on a global scale include vaccinations against human hepatitis B virus and human papilloma viruses, limiting excessive sun exposure, avoiding occupational and environmental carcinogens and measures aimed at tackling alcohol consumption, obesity and physical inactivity (10). Measures to combat some Region-specific risks, including transmission of hepatitis $\mathrm{C}$ virus and schistosomiasis, are also important. In many instances preventive action at the individual level needs to be complemented by legislation and regulation, as has been demonstrated by successes in the case of tobacco control (11).

Despite our knowledge about carcinogenic risks there remain a number of common cancers for which the causes are still poorly understood (e.g. prostate, pancreas, kidney, brain and haematological cancers). Further research here is merited and could make use of geographical and temporal variations in cancer patterns or exposures to throw fresh light on risk factors. Examples from the EMR countries include: the causes of the relatively high rates of lymphoma; the impact of air pollution from natural as opposed to man-made sources; the proportion of liver cancer attributable to hepatitis $\mathrm{B}$ and $\mathrm{C}$; the risks associated with waterpipe smoking; and evaluation of exposure to depleted uranium. Research studies should seek to utilize the exciting new knowledge and associated laboratory tools developed from studying the molecular and cellular underpinnings of cancer towards a better understanding of both causes and prevention (12).

Tackling questions of diet, obesity and physical inactivity should be priorities for countries of EMR. Certainly the development of adapted, standardized dietary surveillance tools to study nutritional transitions in these countries would provide much-needed support to address the epidemic of obesity in both adults and children. In addition, the implementation and evaluation of programmes and policies on diet and obesity, adapted to the Region, need to be prioritized. The etiology of breast cancer, for instance, is dominated by risk factors such as late childbearing and low parity that are related to economic transition and that are less amenable to prevention. Nevertheless, overweight, obesity and lack of physical activity are important and avoidable risk factors in postmenopausal women and should be addressed.

The long natural history of cancer and identifiable pre-cancerous lesions in some tumour types offer opportunities for secondary cancer prevention through early detection, including screening. Effective screening depends on having a national, organized programme. Screening tests, which are just one component of a successful programme, are available for cancers of the cervix, breast, colorectum and oral cavity (in high-risk subjects). There can be no higher priority for secondary cancer prevention in the EMR than identifying effective approaches to the early detection of breast cancer, coupled with adequate treatment and follow-up care.

There often remain barriers to implementation of preventive interventions, with a gap between demonstration of effectiveness in clinical or community-randomized trials and implementation in everyday health care. Lessons come from cervical and breast cancer screening programmes in Latin America, which have failed to provide tangible benefits despite considerable investment $(13,14)$. In response, there is a clear need for implementation of operational research, making good use of pilot or demonstration studies, to assess the barriers (structural, social, professional) to successful cancer control measures. In addition, interventions are often implemented without ongoing evaluation. This is a missed opportunity. First, because inclusion of a research component into a national programme will provide vital information to improve cancer control programmes; secondly, because the cost of research, where national programmes are already funded, is relatively low; and thirdly, because it provides an opportunity to build national research capacity in areas that are close to policy.

In summary, cancer is a complex set of heterogeneous diseases, with remarkable variation in distribution, risk factors, biology, pathology, detection, diagnosis and treatment. Although important cancer control initiatives, particularly in the domain of primary prevention, come through the WHO Global Action Plan for the Prevention and Control of Noncommunicable Diseases 2013-2020, the requirements for comprehensive cancer control in tune with national situations and priorities, especially in the domain of early detection and treatment, demand more focussed inputs (15). One has only to look at the overwhelming prominence of breast cancer in the EMR countries and at the need for access to specialized tertiary-care centres for cancer therapy to realize that a national cancer control plan must stretch beyond the core elements of the WHO noncommunicable diseases agenda. Only in this way will an adequate response emerge to reduce the suffering associated with cancer worldwide.

\section{Acknowledgements}

The author would like to thank Drs Bray, Franceschi and Sankaranarayanan for their helpful comments and also all colleagues from $\mathrm{WHO} /$ EMRO who provided stimulating discussion at the Regional meeting on Cancer Control and Research Priorities in the Eastern Mediterranean Region held in Doha, Qatar, from 20 to 22 October 2013. 


\section{References}

1. Stewart BW, Wild CP. World Cancer Report 2014. Lyon, France: International Agency for Research on Cancer; 2014.

2. GLOBOCAN 2012: estimated cancer incidence, mortality and prevalence worldwide in 2012 [Internet]. Lyon, France: International Agency for Research on Cancer; 2012 (http:// globocan.iarc.fr/Pages/fact_sheets_cancer.aspx, accessed 21 March 2014).

3. Soerjomataram I, Lortet-Tieulent J, Parkin DM, Ferlay J, Mathers C, Forman D, et al. Global burden of cancer in 2008: a systematic analysis of disability-adjusted life-years in 12 world regions. Lancet. 2012 Nov 24;380(9856):1840-50. PMID:23079588

4. Sankaranarayanan R, Swaminathan R, Brenner H, Chen K, Chia KS, Chen JG, et al. Cancer survival in Africa, Asia, and Central America: a population-based study. Lancet Oncol. 2010 Feb;11(2):165-73. PMID:20005175

5. Alwan A. Global status report on noncommunicable diseases 2010. Geneva: World Health Organization; 2011.

6. Mokdad AH, Jaber S, Aziz MI, AlBuhairan F, AlGhaithi A, AlHamad NM, et al. The state of health in the Arab world, 1990-2010: an analysis of the burden of diseases, injuries, and risk factors. Lancet. 2014 Jan 25;383(9914):309-20. PMID:24452042

7. De Martel C, Ferlay J, Franceschi S, Vignat J, Bray F, Forman $D$, et al. Global burden of cancers attributable to infections in 2008: a review and synthetic analysis. Lancet Oncol. 2012 Jun;13(6):607-15. PMID:22575588

8. Global Initiative for Cancer Registry Development (GICR) [Internet]. Lyon, France: International Agency for Research on Cancer; 2014 (http://gicr.iarc.fr, accessed 21 March 2014).
9. Bray F, Znaor A, Cueva P, Korir A, Swaminathan R, Ullrich $A$, et al. Planning and developing population-based cancer registration in low- or middle-income settings. IARC Technical Publication No. 42. Lyon, France: International Agency for Research on Cancer (http://www.iarc.fr/en/publications/ pdfs-online/treport-pub/treport-pub43/index.php accessed 28 May 2014).

10. Vineis P, Wild CP. Global cancer patterns: causes and prevention. Lancet. 2014 Feb 8;383(9916):549-57. PMID:24351322

11. Chaloupka FJ, Straif K, Leon ME; Working Group, International Agency for Research on Cancer. Effectiveness of tax and price policies in tobacco control. Tob Control. 2011 May;20(3):2358. PMID:21115556

12. Wild CP. The exposome: from concept to utility. Int J Epidemiol. 2012 Feb;41(1):24-32. PMID:22296988

13. Murillo R, Almonte M, Pereira A, Ferrer E, Gamboa OA, Jerónimo J, et al. Cervical cancer screening programs in Latin America and the Caribbean. Vaccine. 2008;26 Suppl 11:L37-L48. PMID:18945401

14. Lee BL, Liedke PE, Barrios CH, Simon SD, Finkelstein DM, Goss PE. Breast cancer in Brazil: present status and future goals. Lancet Oncol. 2012;13:e95-e102. PMID:22381937

15. Wild CP. The role of cancer research in noncommunicable disease control. J Natl Cancer Inst. 2012 Jul 18;104(14):1051-8. PMID:22781435 